\title{
Correction to: A Review of Pandemics
}

\author{
Lorenz Hilfiker and Shashwat Ganguly
}

\section{Correction to:}

Chapter 2 in: M. K. Goyal, A. K. Gupta (eds.), Integrated

Risk of Pandemic: Covid-19 Impacts, Resilience and

Recommendations, Disaster Resilience and Green Growth, https://doi.org/10.1007/978-981-15-7679-9_2

Chapter 2 of the book was inadvertently published with incorrect affiliation of the author Lorenz Hilfiker. This has now been corrected. 